\title{
Arithmetic of Unicritical Polynomial Maps
}

\author{
J. Milnor
}

This note will study complex polynomial maps of degree $n \geq 2$ with only one critical point. Such maps can always be put in the standard normal form

$$
f_{c}(z)=z^{n}+c
$$

by an affine change of coordinate. The connectedness locus, consisting of all $c$ for which the Julia set of $f_{c}$ is connected, is sometimes known as the "multibrot set". (Compare $\mathbf{S}$.) It is not difficult to check that the power $\widehat{c}=c^{n-1}$ is a complete invariant for the holomorphic conjugacy class of $f_{c}$.

In $₫ 1$ we will use the alternate normal form

$$
w \mapsto g_{b}(w)=\left(w^{n}+b\right) / n,
$$

with derivative $g_{b}^{\prime}(w)=w^{n-1}$, and use the conjugacy invariant $\widehat{b}=b^{n-1}$. These normal forms (0.1) and (0.2) are related by the change of variable formula

$$
w=n^{1 /(n-1)} z \quad \text { with } \quad \widehat{b}=n^{n} \widehat{c},
$$

and hence $b=n^{n /(n-1)} c$. (In particular, in the degree two case, $b=\widehat{b}$ is equal to $4 c=4 \widehat{c}$.

If $A$ is any ring contained in the complex numbers $\mathbb{C}$, it will be convenient to use the non-standard notation $\bar{A}$ for the integral closure, the ring consisting of all complex numbers which satisfy a monic polynomial equation with coefficients in $A$. (See for example $\mathbf{A M}$.)

Section 1 consists of statements about periodic orbits, which are proved in $₫ 2$ The last section discusses the critically finite case.

\section{Periodic Orbits.}

The following statement generalizes Bousch $\mathbf{B o}$.

THEOREM 1.1. If $w$ is a periodic point for the map $g_{b}$, with multiplier $\mu$, then the rings $\mathbb{Z}[\mu], \mathbb{Z}[w], \mathbb{Z}[b]$, and $\mathbb{Z}[\widehat{b}]$ all have the same integral closure.

Here are some immediate consequences:

COROLlary 1.2. If any one of the four numbers $\mu, w, b, \widehat{b}$ belongs to the ring $\overline{\mathbb{Z}}$ consisting of all algebraic integers, then all four of these numbers are algebraic integers. As an example, if the map $g_{b}$ is parabolic, that is if the multiplier of some periodic orbit is a root of unity, then the parameters $b$ and $\widehat{b}$ are algebraic integers, hence every periodic point $w$ is an algebraic integer, and the multiplier $\mu$ of every periodic orbit is an algebraic integer.

(For a sharper version of this statement, see Remark 2.2.)

I want to thank Thierry Bousch for his help with this manuscript, and the NSF for its support under grant DMSO757856. 
REMARK 1.3. It would be interesting to understand more generally which rational maps have the property that all multipliers are algebraic integers. The family of Lattès maps provides one well known collection of non-polynomial examples.

More generally, if $f: \mathbb{C} \rightarrow \mathbb{C}$ is any polynomial map with only one critical point, then we have the following.

COROLlary 1.4. If $\mu$ and $\mu^{\prime}$ are the multipliers of two periodic orbits for $f$, then the integral closure $\overline{\mathbb{Z}[\mu]}$ is equal to $\overline{\mathbb{Z}\left[\mu^{\prime}\right]}$. It follows that this integral closure depends only on the holomorphic conjugacy class of $f$ and not on the particular choice of periodic orbit.

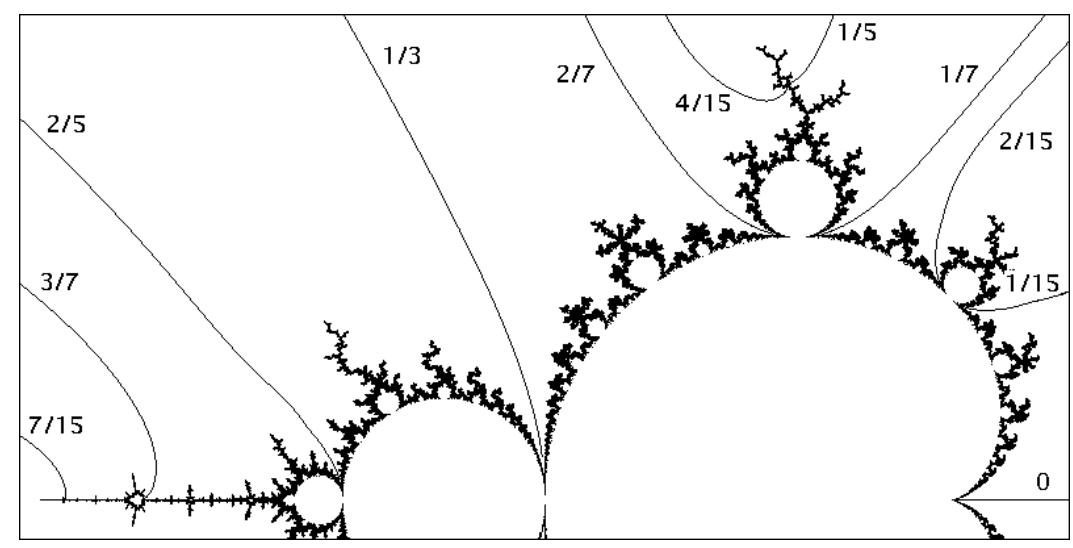

Figure 1. Parameter plane for quadratic maps, showing the external rays in the upper half plane which have period at most four under doubling.

Now suppose that the parameter value $b$ is the landing point of an external ray to the connectedness locus in the $b$-parameter plane, with angle $p / q \in \mathbb{Q} / \mathbb{Z}$ which is periodic under multiplication by $n$. (See Figure 1 for the degree two case.) Then the associated map $g_{b}$ has a parabolic orbit (compare [ES], as well as $[\mathbf{D H}],[\mathbf{L S}$, [M2], [S] ), hence the invariant $\widehat{b}=b^{n-1}=n^{n} \widehat{c}$ is an algebraic integer by Corollary 1.2. We will write $b=b(p / q)$, or $b^{n-1}=\widehat{b}(p / q)$.

There is a curious relationship between the denominator $q$ of this angle and the parameter value $b$ or $\widehat{b}$. Here are some examples in the quadratic case $n=2$, as illustrated in Figure 1, For the landing points of the $1 / 3,2 / 5$, and $3 / 7$-th rays we find that

$$
b(1 / 3)=-3, \quad b(2 / 5)=-5, \quad b(3 / 7)=-7 .
$$

At first glance, this relationship between angles and landing points seems to disappear for the landing point of the $1 / 7$-th and $2 / 7$ rays, with $b=(-1+3 i \sqrt{3}) / 2$. However, this number satisfies the irreducible monic equation

$$
b^{2}+b+7=0,
$$

so the denominator $7=2^{3}-1$ again appears in the description of the landing point. 
Here is a more general statement, working in the $b$ parameter plane for polynomials of degree $n$. If $b$ has degree $d$ over the rational numbers $\mathbb{Q}$, define $\operatorname{Norm}(b) \in \mathbb{Q}$ to be the product of the $d$ algebraic conjugates of $b$ over $\mathbb{Q}$. Up to sign, this is just the constant term in the irreducible monic polynomial satisfied by $b$. If $b$ belongs to the ring $\overline{\mathbb{Z}}$ of algebraic integers, note that $\operatorname{Norm}(b) \in \mathbb{Z}$.

TheOrem 1.5. Consider an external ray of angle $p / q$ in this parameter plane with landing point $b=b(p / q)$. If $p / q$ is periodic under multiplication by $n$ with period $r$, so that $q$ divides $n^{r}-1$, and if $b$ has degree $d$ over $\mathbb{Q}$, then it follows that the integer $\operatorname{Norm}(b)$ is a divisor of $\left(n^{r}-1\right)^{d}$. Similarly, if $\widehat{b}$ has degree $\widehat{d}$ over $\mathbb{Q}$, then $\operatorname{Norm}(\widehat{b})$ divides $\left(n^{r}-1\right)^{(n-1) \widehat{d}}$.

Here are some examples:

- For the landing point of the $1 / 7$-th ray with ray period $r=3$ we have $\operatorname{Norm}(b)=b=-7=-\left(2^{3}-1\right)$

- For the $1 / 5$-th ray, the ray period is four, and the irreducible equation is $b^{3}+9 b^{2}+27 b+135=0$ of degree three with $|\operatorname{Norm}(b)|=135=3^{3} \cdot 5$, which is a divisor of $\left(2^{4}-1\right)^{3}$.

- For an arbitrary degree $n \geq 2$, let $b$ be a fixed point of multiplier $\mu$. Then the equations $g_{b}^{\prime}(w)=\mu$ and $g_{b}(w)=w$ imply that $w^{n-1}=\mu$ and $b=(n-\mu) w$, so that $\widehat{b}=\mu(n-\mu)^{n-1}$. For $\mu=1$, with $r=1$ and $\widehat{d}=1$, it follows that $\widehat{b}=(n-1)^{n-1}$ is precisely equal to $\left(n^{r}-1\right)^{(n-1) \widehat{d}}$. In the case $\mu=-1$, with ray period $r=2$ and $\widehat{d}=1$, we get $\widehat{b}=-(n+1)^{n-1}$, which divides $\left(n^{2}-1\right)^{n-1}$.

\section{Proofs.}

The proofs of the statements of $\$ 1$ will depend on some basic properties of the integral closure. Let $u$ and $v$ be complex numbers. Then clearly $\overline{\mathbb{Z}[u]} \subset \overline{\mathbb{Z}[v]}$ if and only if $u \in \overline{\mathbb{Z}[v]}$. Note also that $\overline{\mathbb{Z}\left[u^{k}\right]}=\overline{\mathbb{Z}[u]}$ for any integer $k>0$. The following statement will be needed.

Lemma 2.1. Let $u$ and $v$ be complex numbers. If the integral closure $\overline{\mathbb{Z}[u]}$ is equal to $\overline{\mathbb{Z}[v]}$, then it is also equal to $\overline{\mathbb{Z}[u v]}$.

Proof. The product $u v$ certainly belongs to the ring $\overline{\mathbb{Z}[u]}=\overline{\mathbb{Z}[v]}$, hence $\overline{\mathbb{Z}[u v]} \subset \overline{\mathbb{Z}[u]}$. Conversely, since $u$ is an element of $\overline{\mathbb{Z}[v]}$, it satisfies an equation of the form

$$
u^{k}=\sum_{i=0}^{k-1} \sum_{j=0}^{\ell} n_{i, j} u^{i} v^{j} \quad \text { with } \quad n_{i, j} \in \mathbb{Z} .
$$

Multiplying both sides of this equation by $u^{\ell}$, the result can be written as

$$
u^{\ell+k}=\sum_{i=0}^{k-1} \sum_{j=0}^{\ell} n_{i, j} u^{\ell+i-j}(u v)^{j},
$$

which proves that $u \in \overline{\mathbb{Z}[u v]}$ hence $\overline{\mathbb{Z}[u]} \subset \overline{\mathbb{Z}[u v]}$. 
Proof of Theorem 1.1. We can write the $k$-fold iterate $g_{b}^{\circ k}(w)$ as a polynomial with integer coefficients divided by a common denominator as follows. Set

$g_{b}^{\circ k}(w)=P_{k}(b, w) / N_{k}$
where $N_{k}=n^{1+n+n^{2}+\cdots+n^{k-1}}=n N_{k-1}^{n}$. Then
$P_{1}(b, w)=w^{n}+b$,

and a straightforward induction shows that

$$
P_{k+1}(b, w)=P_{k}(b, w)^{n}+N_{k}^{n} b .
$$

It follows easily that $P_{k}(b, w)$ is a polynomial in two variables with integer coefficients, and that $P_{k}(b, w)$ is monic of degree $n^{k}$ when considered as a polynomial in $w$ with coefficients in $\mathbb{Z}[b]$, or monic of degree $n^{k-1}$ when considered as a polynomial in $b$ with coefficients in $\mathbb{Z}[w]$.

Now suppose that $w$ is a periodic point for $g_{b}$, with period $h$. Then

$$
g_{b}^{\circ h}(w)-w=0, \quad \text { or in other words } \quad P_{h}(b, w)-N_{h} w=0 .
$$

This last polynomial equation is also monic in either $w$ or $b$, so it follows that $w \in \overline{\mathbb{Z}[b]}$ and that $b \in \overline{\mathbb{Z}[w]}$. Thus the two rings $\mathbb{Z}[b]$ and $\mathbb{Z}[w]$ have the same integral closure. It follows that the ring $\mathbb{Z}[\widehat{b}]$ has this same integral closure.

Now let

$$
w=w_{0} \mapsto w_{1} \mapsto \cdots \mapsto w_{h}=w_{0}
$$

be any period $h$ orbit for $g_{b}$. We know from the argument above that the rings $\mathbb{Z}\left[w_{j}\right]$ all have the same integral closure. It then follows inductively from Lemma 2.1 that the ring $\mathbb{Z}\left[w_{1} w_{2} \cdots w_{h}\right]$ has the same integral closure. Since the multiplier of this orbit can be written as $\mu=\left(w_{1} \cdots w_{h}\right)^{n-1}$, it follows that $\mathbb{Z}[\mu]$ also has the same integral closure.

REMARK 2.2. Here is a supplementary statement. By definition, an element $w \in \overline{\mathbb{Z}}$ is relatively prime to $n$ if the ideal $w \overline{\mathbb{Z}}+n \overline{\mathbb{Z}}$ is equal to $\mathbb{Z}$, or in other words if $w$ maps to a unit in the quotient ring $\overline{\mathbb{Z}} / n \overline{\mathbb{Z}}$. Now suppose that $w \in \overline{\mathbb{Z}}$ is periodic with multiplier $\mu$ under the map $g_{b}$. If any one of the four numbers $w, \mu, b, \widehat{b}$ is prime to $n$, then it follows that all four of these numbers are prime to $n$. As an example, if $g_{b}$ has a parabolic orbit then all of these numbers are prime to $n$, but if $g_{b}$ is critically periodic then none of them is prime to $n$.

To prove this statement, consider an orbit $\left\{w_{j}\right\}$ of period $h$. Then $n w_{j+1}=$ $w_{j}^{n}+b$, hence $w_{j}^{n} \equiv-b(\bmod n \overline{\mathbb{Z}})$. Taking the product over the orbit elements and raising to the $(n-1)$-st power, this yields $\mu^{n} \equiv(-b)^{(n-1) h}$, and the conclusion follows easily.

Proof of Theorem 1.5. Suppose again that $\left\{w_{1}, \ldots, w_{h}\right\}$ is an orbit of period $h$ for $g_{b}$, with multiplier $\mu=u^{n-1}$ where $u=w_{1} w_{2} \cdots w_{h}$. Then we have the congruence

$$
n w_{j+1}=w_{j}^{n}+b \equiv w_{j}^{n} \quad(\bmod b \overline{\mathbb{Z}}) .
$$

In the situation of Corollary 1.2 where $b$ and the $w_{j}$ belong to the ring $\overline{\mathbb{Z}}$ of algebraic integers, we can take the product over $j$ to obtain

$$
n^{h} u \equiv u^{n} \quad(\bmod b \overline{\mathbb{Z}}) .
$$


If $\mu$, and hence $u$, is a unit in the ring $\overline{\mathbb{Z}}$, we can divide this congruence by $u$, yielding

$$
n^{h} \equiv u^{n-1}=\mu \quad(\bmod b \overline{\mathbb{Z}}) .
$$

Now suppose that $\mu$ is a primitive $m$-th root of unity. Then raising this congruence to the $m$-th power, we obtain

$$
n^{h m} \equiv 1 \quad(\bmod b \overline{\mathbb{Z}}) .
$$

Here the product $h m$ is precisely the smallest integer $r$ such that the iterate $g_{b}^{\text {or }}$ maps each $w_{j}$ to itself and has derivative +1 at each $w_{j}$. If an external ray of angle $p / q$ in the $w$-plane lands on $w_{j}$, then $r$ is precisely equal to the ray period, that is the period of $p / q$ under multiplication by $n$. (See for example M2.) Using the usual Douady-Hubbard correspondence between parameter plane and dynamic plane, at least one of these $p / q$ is also the angle of an external ray in the parameter plane which lands on $b$. (Compare $\left[\mathbf{L S}\right.$.) Thus we see that the ratio $\left(n^{r}-1\right) / b$ is an algebraic integer.

Now taking the product over the $d$ distinct embeddings of the field $\mathbb{Q}(b)$ into $\mathbb{C}$, we see that the rational number

$$
\left(n^{r}-1\right)^{d} / \operatorname{Norm}(b)
$$

belongs to $\overline{\mathbb{Z}}$, and hence belongs to the ring $\overline{\mathbb{Z}} \cap \mathbb{Q}=\mathbb{Z}$. In other words, the integer $\operatorname{Norm}(b)$ is a divisor of $\left(n^{r}-1\right)^{d}$, as asserted. A completely analogous argument proves the corresponding statement for $\operatorname{Norm}(\widehat{b})$.

\section{Postcritically Finite Maps.}

The situation for parameter values corresponding to postcritically finite maps is rather different. In this case, it is more convenient to work with the classical normal form of Equation (0.1), with invariant $\widehat{c}=c^{n-1}=\widehat{b} / n^{n}$. The analogue of Corollary 1.2 in this context is the following.

LEMma 3.1. If $z$ is periodic under $f_{c}$, then $z \in \overline{\mathbb{Z}}$ if and only if the parameter $c$ or $\widehat{c}$ belongs to $\overline{\mathbb{Z}}$. In this case, the multiplier $\mu$ of the orbit belongs to the ideal $n^{h} \overline{\mathbb{Z}}$, where $h$ is the period.

The proof is not difficult. (Compare Remark 3.3.)

In the critically periodic case, it is not hard to show that $c \in \overline{\mathbb{Z}}$. This statement can be sharpened as follows for $c \neq 0$.

THEOREM 3.2. If the orbit of the critical point is eventually periodic, then $c$ and $\widehat{c}=c^{n-1}$ are algebraic integers, with $\operatorname{Norm}(\widehat{c})$ dividing $n$. In the special case where the critical point is actually periodic with period $>1$, we can sharpen this statement to say that $\operatorname{Norm}(\widehat{c})= \pm 1$.

Here are some quadratic examples. If $c=-1$ then the critical point has period 2 , while if $c^{3}+2 c^{2}+c+1=0$ it has period 3 . A number of critically preperiodic cases are shown in Figure 2, and described further in Tables 1 and 2 (Here the transient time is defined to be the smallest $t$ such that $f_{c}^{\circ t}(c)$ is periodic.) Note that there is no evident arithmetic relation between the external angles and the landing point $c$ in these cases. 


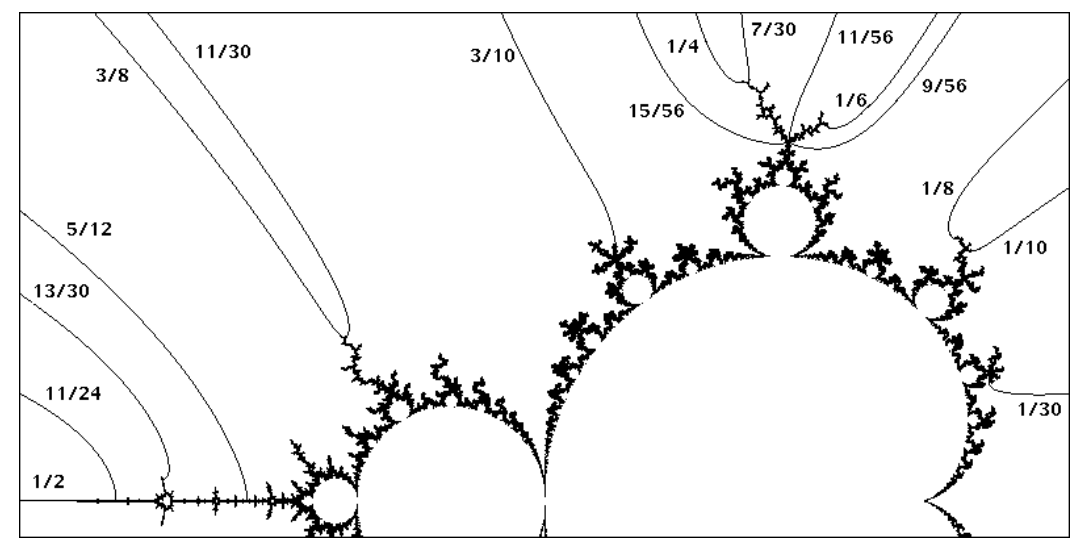

Figure 2. Some eventually periodic rays for the Mandelbrot set.

\begin{tabular}{|ccccc|}
\hline$p / q$ & transient time & eventual period & degree $(c)$ & $\mid$ Norm $(c) \mid$ \\
\hline$\frac{1}{2}$ & $t=1$ & $h=1$ & $d=1$ & 2 \\
$\frac{1}{6}$ & $t=1$ & $h=2$ & $d=2$ & 1 \\
$\frac{1}{4}, \frac{5}{12}$ & $t=2$ & $h=1$ & $d=3$ & 2 \\
$\frac{1}{8}, \frac{9}{56}, \frac{11}{56}, \frac{15}{56}, \frac{3}{8}, \frac{11}{24}$ & $t=3$ & $h=1$ & $d=7$ & 2 \\
$\frac{1}{30}, \frac{1}{10}, \frac{7}{30}, \frac{3}{10}, \frac{11}{30}, \frac{13}{30}$ & $t=1$ & $h=4$ & $d=12$ & 1 \\
\hline
\end{tabular}

TABLE 1. Description of the corresponding landing points $c$.

$c+2=0$
$c^{2}+1=0$
$c^{3}+2 c^{2}+2 c+2=0$
$c^{7}+4 c^{6}+6 c^{5}+6 c^{4}+6 c^{3}+4 c^{2}+2 c+2=0$
$c^{12}+6 c^{11}+15 c^{10}+22 c^{9}+23 c^{8}+18 c^{7}+11 c^{6}+8 c^{5}+6 c^{4}+2 c^{3}+1=0$.

TABLE 2. Corresponding irreducible equations.

Note that there can be many different postcritically finite parameters which satisfy the same irreducible equation over $\mathbb{Q}$. This is related to the fact that the Galois group of $\overline{\mathbb{Q}}$ over $\mathbb{Q}$ may act in a highly non-trivial way on these points. (Compare [P], as well as Remark 3.5])

Proof of Theorem 3.2. Since $c \neq 0$, we can use the modified normal form $\zeta \mapsto F_{\widehat{c}}(\zeta)$ where

$$
F_{\widehat{c}}(\zeta)=\frac{f_{c}(c \zeta)}{c}=\frac{(c \zeta)^{n}+c}{c}=\widehat{c} \zeta^{n}+1,
$$


with critical orbit of the form

$$
0 \mapsto 1 \mapsto \widehat{c}+1 \mapsto \cdots .
$$

The $k$-th point of this critical orbit can be expressed as a polynomial function $P_{k}(\widehat{c})$, with $P_{1}=1$ and

$$
P_{k+1}(\widehat{c})=\widehat{c} P_{k}(\widehat{c})^{n}+1 \text {. }
$$

Evidently each $P_{k}(\widehat{c})$ is a monic polynomial with constant term $P_{k}(0)=+1$. Therefore, if $F_{\widehat{c}}$ has periodic critical point, then it follows that $\widehat{c}$ is a unit in the ring of algebraic integers, with $\operatorname{Norm}(\widehat{c})= \pm 1$.

Now suppose that the orbit of zero is eventually periodic but not periodic. Then the transient time $t \geq 1$, and the eventual period $h \geq 1$ are defined as the smallest positive integers such that $F_{\widehat{c}}^{\circ t}(1)$ is periodic of period $h$. It follows that the two orbit points $P_{t}(\widehat{c})$ and $P_{t+p}(\widehat{c})$ are distinct, and yet have the same image under the $n$-th power map. In other words the ratio

$$
x=P_{t+p}(\widehat{c}) / P_{t}(\widehat{c})
$$

must be an $n$-th root of unity, not equal to +1 . Hence it must satisfy the equation

$$
1+x+x^{2}+\cdots+x^{n-1}=0 .
$$

Clearing denominators, we see that

$$
\sum_{i+j=n-1} P_{t+p}(\widehat{c})^{i} P_{t}(\widehat{c})^{j}=0 .
$$

It is not difficult to check that this is a monic polynomial equation in $\widehat{c}$ with constant term $n$. Therefore $\widehat{c}$ is an algebraic integer, and $\operatorname{Norm}(\widehat{c})$ divides $n$.

REMARK 3.3. Note that any periodic point for the map $f_{c}$ satisfies a monic equation $f_{c}^{\circ h}(z)-z=0$ with coefficients in $\mathbb{Z}[c]$. Whenever $c \in \overline{\mathbb{Z}}$ and hence $\mathbb{Z}[c] \subset \overline{\mathbb{Z}}$, it follows that $z \in \overline{\mathbb{Z}}$, hence $f_{c}^{\prime}(z) \in n \overline{\mathbb{Z}}$, so that the multiplier $\mu$ belongs to the ideal $n^{h} \overline{\mathbb{Z}}$. In fact there seems to be a strong tendency for periodic points to be units in the ring $\overline{\mathbb{Z}}$, so that the ratio $\mu / n^{h}$ is also a unit. As an example, suppose that $\widehat{c}=-1$ so that the critical orbit has period two. Then the equation $f_{c}^{\circ h}(z)-z=0$ is monic with constant term $c=(-1)^{1 /(n-1)}$ when $h$ is odd, and the ratio $\left(f_{c}^{\circ h}(z)-z\right) / z$ is monic with constant term -1 when $h$ is even. Hence every periodic point $z \neq 0$ for $f_{c}$ is an algebraic unit.

REMARK 3.4. Let $\left\{z_{j}\right\}$ be a periodic orbit of period $h>1$ so that

$$
z_{j+1}=z_{j}^{n}+c
$$

where $j$ ranges over $\mathbb{Z} / h \mathbb{Z}$. Using the polynomial expression

$$
\phi(x, y)=\frac{x^{n}-y^{n}}{x-y}=x^{n-1}+x^{n-2} y+\cdots+x y^{n-2}+y^{n-1},
$$

note the identity

$$
\frac{z_{j+2}-z_{j+1}}{z_{j+1}-z_{j}}=\frac{z_{j+1}^{n}-z_{j}^{n}}{z_{j+1}-z_{j}}=\phi\left(z_{j}, z_{j+1}\right) .
$$

Taking the product over all $j$ modulo $h$, it follows that $\prod_{j \bmod h} \phi\left(z_{j}, z_{j+1}\right)=1$. (Compare Benedetto $\left[\mathbf{B e}\right.$.) In particular, if $c \in \overline{\mathbb{Z}}$ so that the $z_{j}$ also belong to $\overline{\mathbb{Z}}$, then it follows that each expression $\phi\left(z_{j}, z_{j+1}\right)$ is a unit in the ring $\overline{\mathbb{Z}}$. (For other "dynamical units", see [MS.) 
Remark 3.5 (Classical Problems). To conclude this discussion, we mention two well known unsolved problems.

If the maps $f_{c_{1}}$ and $f_{c_{2}}$ have parabolic orbits with the same period and the same ray period, does it follow that the corresponding invariants $\widehat{c}_{1}$ and $\widehat{c}_{2}$ satisfy the same irreducible equation over $\mathbb{Q}$ ?

In other words, does it follow that $\widehat{c}_{1}$ and $\widehat{c}_{2}$ are conjugate under some Galois automorphism of the field $\overline{\mathbb{Q}}$ over $\mathbb{Q}$ ?

Similarly, if two maps $f_{c_{1}}$ and $f_{c_{2}}$ have critical orbits which are periodic with the same period, does it follow that $\widehat{c}_{1}$ and $\widehat{c}_{2}$ are Galois conjugate?

There is a similar question for the eventually periodic case, but the situation is more complicated. There is an extra invariant if the degree $n$ is not prime, since the ratio of Equation (3.1) above must be a primitive $\tau$-th root of unity for some divisor $\tau$ of $n$, with $1<\tau \leq n$.

If two such parameter values have the same transient time $t$, the same eventual period $h$, and the same integer $1<\tau \mid n$, does it follow that the corresponding invariants $\widehat{c}$ are Galois conjugate?

\section{References}

[AM] M. Atiyah and I. Macdonald, "Introduction to Commutative Algebra", Addison-Wesley 1969.

[Be] R. Benedetto, An elementary product identity in polynomial dynamics, Amer. Math. Monthly 108 (2001) 860-864.

[Bo] T. Bousch, Les racines des composantes hyperboliques de $M$ sont des quarts d'entiers algébriques, (manuscript, 1996). To appear in "Frontiers in Complex Dynamics: a volume in honor of John Milnor's 80th birthday," A. Bonifant, M. Lyubich, S. Sutherland, editors. Princeton University Press 2012.

[DH] A. Douady and J. H. Hubbard, "Étude dynamique des polynômes complexes I \& II", Publ. Math. Orsay (1984-85)

[LS] E. Lau and D. Schleicher, Internal addresses in the Mandelbrot set and irreducibility of polynomials, Stony Brook IMS Preprint 1994/19.

[ES] D. Eberlein and D. Schleicher, Rational parameter rays of multibrot sets, in preparation.

[M1] J. Milnor, "Dynamics in One Complex Variable", Princeton U. Press 2006.

[M2] J. Milnor, Periodic Orbits, Externals Rays and the Mandelbrot Set: An Expository Account, In "Geometrie Complexe et Systemes Dynamiques," ed. M. Flexor, P. Sentenac, J.C. Yoccoz, Astérisque 261 (2000) 277-333.

[MS] P. Morton and J. Silverman, Periodic points, multiplicities, and dynamical units, J. Reine Angew. Math. 461 (1995) 81-122.

[P] K. Pilgrim, Dessins d'enfants and Hubbard Trees, Ann. Sci. École Norm. Sup. (4) 33 (2000) 671-693.

[S] D. Schleicher, On fibers and local connectivity of Mandelbrot and Multibrot sets, in "Fractal Geometry and Applications: a jubilee of Benoît Mandelbrot. Part 1," Proc. Sympos. Pure Math., 72, Part 1, Amer. Math. Soc. (2004) 477-517. 
March, 2012

Institute for Mathematical Sciences, Stony Brook University, Stony Brook, Ny 11794-3660

E-mail address: jack@math.sunysb.edu 